\title{
Professional oral health care reduces the duration of hospital stay in patients undergoing orthognathic surgery
}

\author{
HIDEO SHIGEISHI $^{1}$, MOHAMMAD ZESHAAN RAHMAN ${ }^{1}$, KOUJI OHTA ${ }^{1}$, \\ SHIGEHIRO ONO ${ }^{1}$, MASARU SUGIYAMA ${ }^{2}$ and MASAAKI TAKECHI ${ }^{1}$ \\ ${ }^{1}$ Department of Oral and Maxillofacial Surgery; ${ }^{2}$ Public Oral Health, Institute of Biomedical \\ and Health Sciences, Hiroshima University, Hiroshima 734-8553, Japan
}

Received August 26, 2015; Accepted October 2, 2015

DOI: $10.3892 /$ br.2015.542

\begin{abstract}
The present study reviewed the records of 58 patients who underwent orthognathic surgery [sagittal split ramus osteotomy (SSRO), Le Fort I osteotomy, genioplasty, anterior maxillary alveolar osteotomy] between 2010 and 2015. To investigate the influence of preoperative oral health care on postoperative inflammation, infection and length of hospital stay in those patients, white blood cell (WBC) count and C-reactive protein (CRP) levels were compared between patients who received and did not receive preoperative oral care. The mean CRP level throughout the postoperative term was lower in the oral care group as compared to the non-oral care group. By contrast, the oral care group had a lower occurrence of postoperative infectious complications (surgical site infection, anastomotic leak) (13.6 vs. 20.8\%) and a shorter average length of hospital stay (16.2 \pm 3.8 vs. $21.2 \pm 7.4$ days). These results suggest that preoperative professional oral health care decreases the duration of hospital stay following orthognathic surgery by inhibiting inflammation and infectious complications during the postoperative stage.
\end{abstract}

\section{Introduction}

Orthognathic surgery has become a standard procedure in the field of oral and maxillofacial surgery. For mandibular orthognathic surgery, a sagittal split ramus osteotomy (SSRO) is a popular procedure that can be performed on an intraoral basis (1), while a Le Fort I osteotomy is widely applied for

Correspondence to: Dr Hideo Shigeishi, Department of Oral and Maxillofacial Surgery, Institute of Biomedical and Health Sciences, Hiroshima University, 1-2-3 Kasumi, Hiroshima 734-8553, Japan E-mail: shige@hiroshima-u.ac.jp

Abbreviations: SSRO, sagittal split ramus osteotomy; WBC, white blood cell; CRP, C-reactive protein

Key words: oral health care, retrospective study, jaw bone osteotomy removal and accurate repositioning of the maxilla (2). However, despite advances in surgical techniques used for orthognathic surgery, various complications that occur following surgery, including hypoesthesia, bleeding, respiratory difficulty, temporomandibular joint dysfunction and infection, have been documented $(3,4)$. Among those, surgical site infection is an important problem, as it delays wound repair and prolongs hospital stay. In order to prevent wound infection, use of antibiotics as well as application of oral health care procedures, such as professional teeth cleaning and self-care instructions, are considered as important factors for patients undergoing oral surgery, as they are associated with reduced numbers of oral bacteria, resulting in inhibition of surgical site infection in oral surgery cases.

However, few studies have shown a significant association between preoperative oral health care and postoperative complications in patients who underwent orthognathic surgery. In the present study, the influence of preoperative oral health care on postoperative inflammatory response, infection and length of hospital stay was investigated in patients undergoing orthognathic surgery.

\section{Patients and methods}

Patient characteristics. The records of 58 patients (17 males, 41 females; mean age, 25.8 years; range, 16-49 years) with jaw bone deformity who underwent surgical treatment in the surgical room under general anesthesia at the Department of Oral and Maxillofacial Reconstructive Surgery, Hiroshima University Hospital (Hiroshima, Japan), between January 2010 and March 2015 were reviewed. Subjects included in the study were those who underwent an orthognathic surgery procedure, including SSRO ( $\mathrm{n}=42)$, SSRO and Le Fort I osteotomy $(n=13)$, genioplasty $(n=2)$, and anterior maxillary alveolar osteotomy $(n=1)$. All the surgeries were performed intraorally without abnormal bone fracture or blood transfusion. Clinical data obtained included patient age, gender, medical history, blood loss volume and surgical duration. Patients with a past history of diabetes, respiratory disorders, cardiovascular disorders, hyperlipidemia, hypoalbuminemia or others were not included in order to exclude the effects of those diseases on postoperative conditions. Cephem antibiotics (cefdinir or cefmetazole) were administered in all patients for 3-5 days after the surgery. 
Treatment. Patients in the oral care group received professional teeth cleaning or scaling by a dental hygienist within 2 days prior to surgery, while those in the non-oral care group did not receive special treatment from a dental hygienist prior to surgery. Following surgery, regular oral care was performed for all patients in the two groups by a doctor at least once a day.

Markers and statistical analysis. Markers, such as white blood cell (WBC) count and C-reactive protein (CRP) level, were examined on the day before and at 1,3-5, and 7-9 days after surgery to evaluate the inflammatory response following the surgery. Occurrences of complications such as anastomotic leak and surgical site infection were investigated within 14 days after surgery, with the latter determined according to the method of Johnson et al (5). The length of hospital stay for each patient was also examined. Welch's t-test, a one-way analysis of variance test, and Fisher's exact test were used for statistical analysis. $\mathrm{P}<0.05$ was considered to indicate a statistically significant difference. This retrospective study was approved by the ethics committee of Hiroshima University.

\section{Results}

Correlation between postoperative complications and clinical features. The associaton between preoperative oral care and postoperative infectious complications (surgical site infection, aspiration pneumonia, anastomotic leak) was investigated. One case of surgical site infection was observed in the non-oral care group, while an anastomotic leak was identified in 4 (13.8\%) in the non-oral care and 3 (10.3\%) in the oral care group. No aspiration pneumonia was reported in any of the patients. The correlation between postoperative complications and clinical features, such as gender, age, surgical duration, blood loss and pre-operative oral health care (Fisher's exact test), was subsequently examined (Table I). Although there was no statistical association identified between them, the rate of occurrence of complications was decreased in patients who received preoperative oral health care (10.3\%) as compared to those who did $\operatorname{not}(17.2 \%)$.

Comparison of inflammatory response between oral care and non-oral care groups. The preoperative WBC count in the oral care and non-oral care groups was $5,941 / \mathrm{mm}^{3}$ and $5,746 / \mathrm{mm}^{3}$, respectively, both of which were within a normal range. On day 1 after surgery, the counts increased to a maximum of $13,344 / \mathrm{mm}^{3}$ and $13,230 / \mathrm{mm}^{3}$, subsequently decreased to $7,607 / \mathrm{mm}^{3}$ and $6,973 / \mathrm{mm}^{3}$ on days $3-5$, and returned to $6,132 / \mathrm{mm}^{3}$ and $6,155 / \mathrm{mm}^{3}$ on days 7-9, respectively (Fig. 1A). The mean WBC count in the oral care group was lower compared to the non-oral care group at days 3-5 and 7-9 after surgery, but was not significant.

The preoperative CRP level was within a normal range in all the patients in the two groups. The mean postoperative CRP in the non-oral care group increased to a maximum of $5.82 \mathrm{mg} / \mathrm{dl}$ on day 1 and decreased to $1.77 \mathrm{mg} / \mathrm{dl}$ on days 3-5 (Fig. 1B). In the oral care group, the mean post-operative CRP increased to a maximum of $5.21 \mathrm{mg} / \mathrm{dl}$ on day 1 , decreased to $0.87 \mathrm{mg} / \mathrm{dl}$ on days 3-5 and returned to $0.22 \mathrm{mg} / \mathrm{dl}$ on days $7-9$ (Fig. 1B).
A

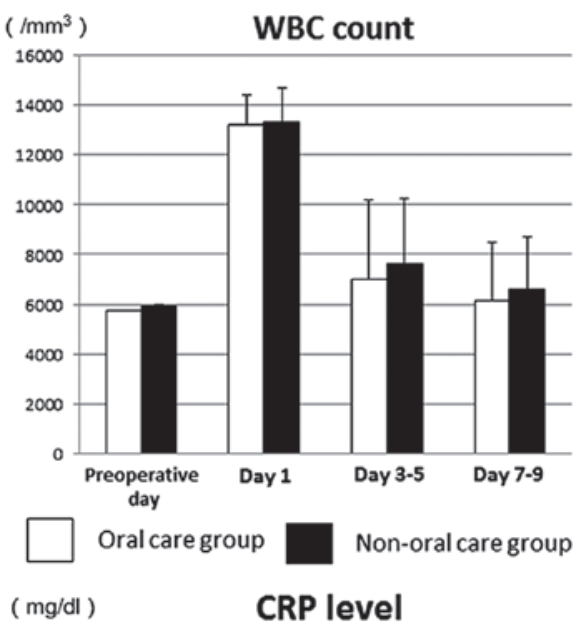

B

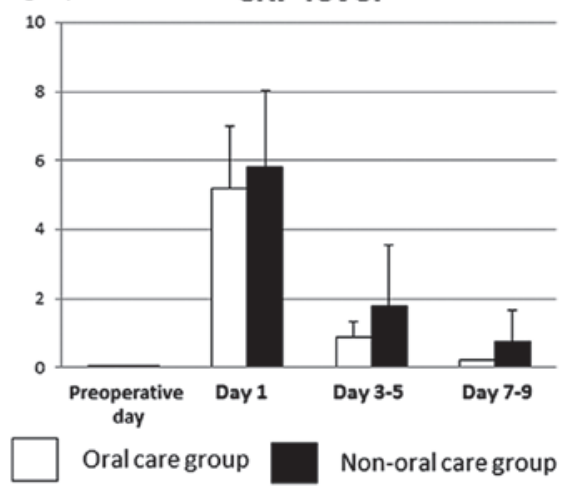

Figure 1. WBC count and CRP level in oral care and non-oral care groups. (A) There was no significant difference in regards to WBC count between the groups. (B) Postoperative mean CRP in the non-oral care group was higher compared to the oral care group on days 1, 3-5 and 7-9, but not statistically significant. Error bars represent the mean \pm standard deviation. WBC, white blood cell count; CRP, C-reactive protein.

The mean CRP in the oral care group was lower compared to the non-oral care group at each time point following surgery, but was not significant. These results suggest that preoperative oral care may aid in inhibiting inflammation in the early postoperative period.

Hospitalization duration. To investigate the effect of preoperative oral health care on postoperative recovery, the length of hospital stay was examined. In the oral care and non-oral care groups, the mean hospital stay was $16.2 \pm 3.8$ and $21.2 \pm 7.4$ days, respectively (Fig. $2 \mathrm{~A}$ ), which was significantly shorter for the patients who received preoperative oral care. In addition, this was significantly shorter in cases with a surgical time $<180 \mathrm{~min}$ as compared to those with a longer surgical time (14.7 \pm 3.8 vs. $19.6 \pm 6.4$ days) (Fig. $2 B$ ). By contrast, the length of hospital stay was not significantly associated with blood loss (Fig. 2C). As for the surgical method, patients who underwent SSRO and Le Fort I osteotomy procedures in combination showed the longest hospital stay duration (21.5 \pm 4.1 days) due to the severity of the procedure (Fig. 2D). However, no significant difference was identified among the surgical procedures employed. These results indicate that preoperative oral health care and surgical time may be associated with recovery of the wound area in the oral cavity in patients who underwent orthognathic surgery. 
Table I. Correlation between postoperative complications and clinical factors.

\begin{tabular}{|c|c|c|c|c|}
\hline \multirow[b]{2}{*}{ Clinical factors } & \multirow[b]{2}{*}{ Cases, $\mathrm{n}$} & \multicolumn{2}{|c|}{$\begin{array}{c}\text { Postoperative } \\
\text { complications, } \\
\mathrm{n}(\%)\end{array}$} & \multirow[b]{2}{*}{ P-value } \\
\hline & & $(-)$ & $(+)$ & \\
\hline \multicolumn{5}{|l|}{ Gender } \\
\hline Male & 17 & $15(88.2)$ & $2(11.8)$ & 1.00 \\
\hline Female & 41 & $35(85.4)$ & $6(14.6)$ & \\
\hline \multicolumn{5}{|l|}{ Age, years } \\
\hline $16-19$ & 17 & $14(85.7)$ & $3(14.3)$ & 0.65 \\
\hline $20-29$ & 23 & $19(82.6)$ & $4(17.4)$ & \\
\hline $30-39$ & 12 & $11(91.7)$ & $1(8.3)$ & \\
\hline $40-49$ & 6 & $0(0.0)$ & $1(100)$ & \\
\hline \multicolumn{5}{|l|}{ Surgical procedure } \\
\hline SSRO & 42 & $38(90.5)$ & $4(9.5)$ & 0.060 \\
\hline SSRO \& Lefort I & 13 & $11(84.6)$ & $2(15.4)$ & \\
\hline Genioplasty & 2 & $1(50.0)$ & $1(50.0)$ & \\
\hline $\begin{array}{l}\text { Maxillary alveolar } \\
\text { osteotomy }\end{array}$ & 1 & $0(0.0)$ & $1(100)$ & \\
\hline \multicolumn{5}{|l|}{ Oral care } \\
\hline$(-)$ & 29 & $24(82.8)$ & $5(17.2)$ & 0.71 \\
\hline$(+)$ & 29 & $26(89.7)$ & $3(10.3)$ & \\
\hline \multicolumn{5}{|l|}{ Blood loss, ml } \\
\hline$<300$ & 18 & $16(88.9)$ & $2(11.1)$ & 0.64 \\
\hline$\geq 300$ & 40 & $34(85.0)$ & $6(15.0)$ & \\
\hline \multicolumn{5}{|l|}{ Surgical time, $\min$} \\
\hline$<180$ & 11 & $9(81.8)$ & $2(18.2)$ & 1.00 \\
\hline$\geq 180$ & 47 & $41(87.2)$ & $6(12.8)$ & \\
\hline
\end{tabular}

\section{Discussion}

Patients who undergo oral surgery often have difficulties with elimination of dental plaque due to surgery-related swelling, bleeding and pain following the surgery. Without regular removal, dental plaque undergoes a process of maturation that results in development of pathogenic bacterial flora. Therefore, proper oral health care during the perioperative period is thought to be necessary to decrease the number of oral pathogens. A previous study showed that oral care is essential to decrease surgical site infection in oral cancer patients (6). In addition, preoperative oral care was shown to reduce inflammation during the early postoperative stage in oral cancer patients who underwent a combination of surgical resection with neck dissection and free-flap transplantation, or pectoralis major myocutaneous flap transplantation (7). Together, these results suggest that oral health care is required for oral cancer patients to prevent wound infection. As those patients have difficulties with cleaning their oral cavity, professional oral hygiene procedures, such as mechanical plaque removal from teeth, gingival and mucosal surfaces, can help them maintain their oral health condition. Furthermore, proper instructions
A
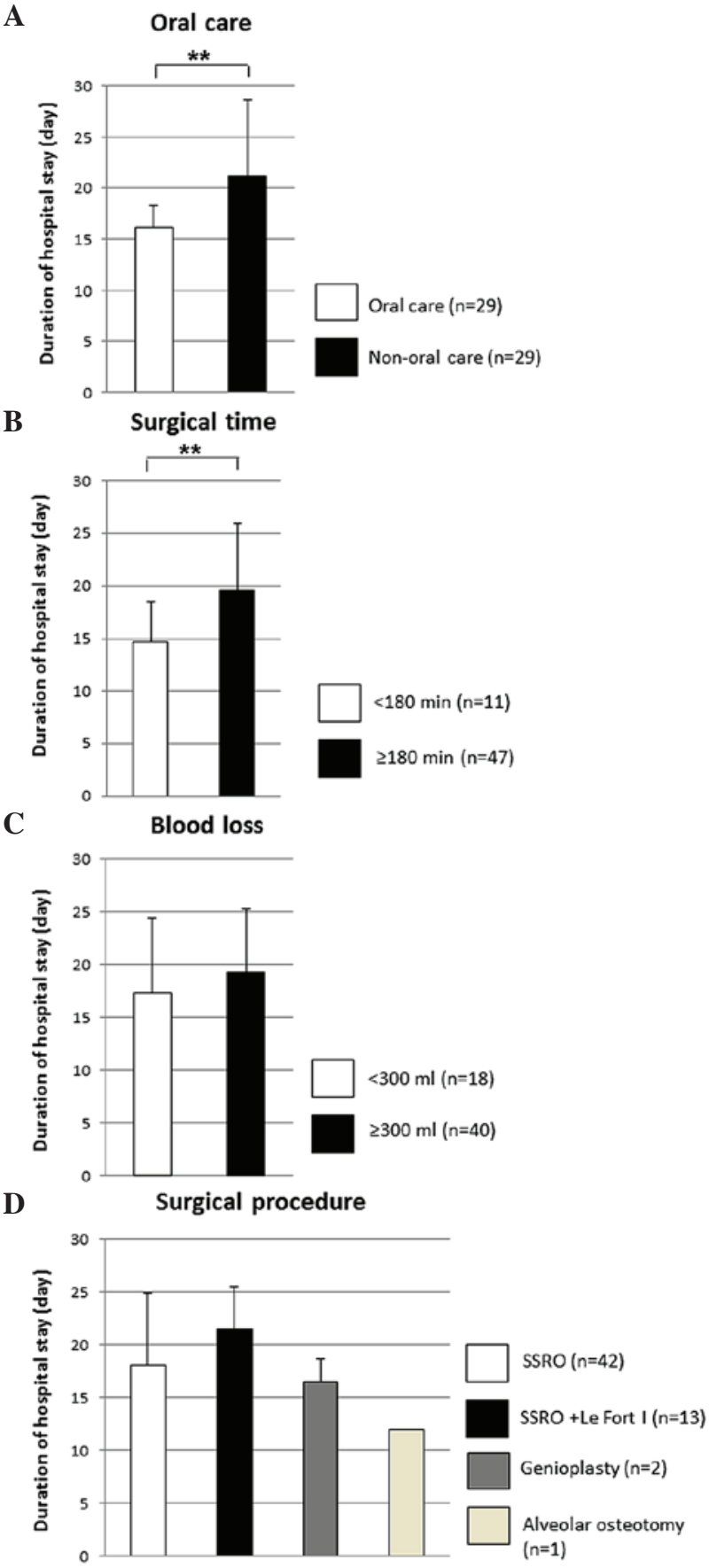

Figure 2. Correlation between duration of hospital stay and clinical factors (A) Mean hospital stay duration was significantly decreased in the oral care group as compared to the non-oral care group ( $\mathrm{P}=0.0022$, Welch's t-test). (B) Mean hospital stay duration was significantly reduced in patients with a surgical time of $<180 \mathrm{~min}$ as compared to those with a longer surgical time ( $\mathrm{P}=0.0032$, Welch's t-test). (C) There was no significant difference for hospital stay duration between patients with $\geq 300 \mathrm{ml}$ of blood loss and those with a smaller amount of blood loss (Welch's t-test). (D) There were no significant differences for hospital stay duration among the surgical procedures employed (one-way analysis of variance). ${ }^{* *} \mathrm{P}<0.01$, statistically significant.

for self-oral care may help the affected patients to maintain good oral health in the postoperative period.

By contrast, few studies have evaluated the effects of preoperative oral health care on postoperative complications in patients who undergo an oral surgery procedure, such as orthognathic surgery. In the present study of the association 
between preoperative oral care and inflammatory response parameters, no significant difference was identified in regard to the percentage of postoperative infectious complications between the oral care $(10.3 \%)$ and non-oral care $(17.2 \%)$ groups, although a declining tendency of postoperative complications following preoperative oral care was noted. Professional oral health care appears to have an important role in managing infectious complications by reducing the number of oral pathogens present at the time of surgery.

As the CRP level can rapidly increase during the acute phase of inflammation (8), it is considered to be a sensitive inflammatory marker and useful indicator for detection of infection following surgery $(9,10)$. Although there were no significant differences in regards to WBC count and CRP level between the present oral care and non-oral care groups, CRP levels were clearly different between the groups on days 1,3-5, and 7-9. One possible reason is that an inflammatory condition in the early postoperative period may be affected by preoperative professional oral health care, and that in the late postoperative period it may be influenced by pre- and postoperative oral health care.

As for the duration of hospital stay, the median duration was significantly lower in the oral care group as compared with the non-oral care group, suggesting that a shortened hospital stay may be attributed to rapid recovery from postoperative inflammation and proper wound healing. Therefore, the present findings suggest that preoperative oral health care can shorten the length of hospital stay by attenuating the occurrence of inflammation and infectious complications during the postoperative stage.

Previous studies have identified a significant association between blood loss and frequency of surgical site infection in patients who underwent head and neck surgery $(6,11,12)$. In the present study, no significant differences in regards to the occurrence of postoperative infectious complications between patients with low blood loss $(11.1 \%)$ and those with high blood loss $(15.0 \%)$ were observed, although postoperative complications showed a declining tendency in the former group. Therefore, blood loss may be a risk factor for infection in patients undergoing orthognathic surgery.

In summary, the present findings indicate that preoperative oral health care may help to reduce postoperative inflammation and the frequency of postoperative complications in patients undergoing orthognathic surgery, resulting in a shorter hospital stay.

\section{Acknowledgements}

The present study was supported by a Grant-in-aid (no. 23592963) from the Japanese Ministry of Education, Culture, Sports and Technology.

\section{References}

1. Al-Moraissi EA and Ellis E III: Is there a difference in stability or neurosensory function between bilateral sagittal split ramus osteotomy and intraoral vertical ramus osteotomy for mandibular setback? J Oral Maxillofac Surg 73: 1360-1371, 2015.

2. de Mol van Otterloo JJ, Tuinzing DB, Greebe RB and van der Kwast WA: Intra- and early postoperative complications of the Le Fort I osteotomy: A retrospective study on 410 cases. J Craniomaxillofac Surg: 19: 217-222, 1991.

3. Garg S and Kaur S: Evaluation of post-operative complication rate of le fort I osteotomy: A retrospective and prospective study. J Maxillofac Oral Surg 13: 120-127, 2014.

4. Mitsukawa N, Morishita T, Saiga A, Kubota Y, Omori N, Akita S and Satoh K: Dislocation of temporomandibular joint: Complication of sagittal split ramus osteotomy. J Craniofac Surg 24: 1674-1675, 2013.

5. Johnson JT, Myers EN, Thearle PB, Sigler BA and Schramm VLJr: Antimicrobial prophylaxis for contaminated head and neck surgery. Laryngoscope 94: 46-51, 1984.

6. Sato J, Goto J, Harahashi A, Murata T, Hata H, Yamazaki Y, Satoh A, Notani K and Kitagawa Y: Oral health care reduces the risk of postoperative surgical site infection in inpatients with oral squamous cell carcinoma. Support Care Cancer 19: 409-416, 2011.

7. Shigeishi H, Ohta K, Fujimoto S, Nakagawa T, Mizuta K, Ono S, et al: Preoperative oral health care reduces postoperative inflammation and complications in oral cancer patients. Exp Ther Med (In press).

8. Pepys MB and Hirschfield GM: C-reactive protein: A critical update. J Clin Invest 111: 1805-1812, 2003.

9. Iizuka $\mathrm{T}$ and Lindqvist $\mathrm{C}$ : Changes in C-reactive protein associated with surgical treatment of mandibular fractures. J Oral Maxillofac Surg 49: 464-467, 1991.

10. Mustard RA Jr, Bohnen JM, Haseeb S and Kasina R: C-reactive protein levels predict postoperative septic complications. Arch Surg 122: 69-73, 1987.

11. Liu SA, Wong YK, Poon CK, Wang CC, Wang CP and Tung KC: Risk factors for wound infection after surgery in primary oral cavity cancer patients. Laryngoscope 117: 166-171, 2007.

12. Ogihara H, Takeuchi K and Majima Y: Risk factors of postoperative infection in head and neck surgery. Auris Nasus Larynx 36: 457-460, 2009. 